\title{
Vibration propagation in discrete element particle models of rock
}

\author{
R. Resende \\ ISCTE-IUL/ADETTI-IUL, Lisbon, Portugal
}

E. Fortunato

National Laboratory for Civil Engineering, Lisbon, Portugal

C. Andrade

Faculty of Engineering of the Porto University, Porto, Portugal

T. Miranda

ISISE, Department of Civil Engineering, University of Minho, Guimarães, Portugal

\begin{abstract}
Vibrations in rock masses can be a significant hazard, leading to human discomfort and structural damage. Recent cases of subway or tunnel excavation near existing dams in Portugal have raised again the importance of this topic. Traditional statistical tools have been used to study wave propagation in rock, but fall short when rock fracturing or faulting radically changes the way vibration propagates. Numerical models such as finite-element or finite-difference models also have some insufficiencies, namely in the way discontinuities are represented. We propose the utilization of bonded particle models based on the Particle Discrete Element Method. Synthetic rock samples are generated and their static elastic properties are calibrated by manipulating microproperties (assembly organization and normal and shear contact stiffness). The static and dynamic properties of the rock cores are tested and the complete elastic response of the model is evaluated and discussed.
\end{abstract}

\section{INTRODUCTION}

Ground vibrations can lead to structural damage, induce equipment failure and hinder human activities. Rock blasting is one of the main generators of vibration, and when the structures, equipment or populations that are affected are on the same rock mass, attenuation is low and vibration levels can be very high.

Standard statistical methods have been used for some decades to address these problems, but fall short in many situations were rock fracturing or faulting radically changes the way vibration propagates. In these situations, numerical models such as finiteelement or finite-difference models also have some insufficiencies, namely in the way rock fractures are represented.

Particle discrete element models can contribute by complementing fundamental studies, as they allow visualization of internal behaviour of rock. On the other hand, it is very likely that, with the advent of powerful hardware and adequate codes, we will be able to perform real scale calculations with particle models in the near future.

This work (Andrade 2012) investigates the generation and calibration process of discrete element particle models with well-defined static and dynamic properties. In this context, synthetic rock samples are generated and their static elastic properties are calibrated by manipulating micro-properties (assembly organization and shear contact stiffness). Then the dynamic properties of the rock cores are tested through the simulation of shear and compression wave velocity tests.

We are able to develop empirical relations between static and dynamic micro and macro properties, rendering the process of static and dynamic model construction more evident and easy and also relate the emergent dynamic behaviour of the model to that of real rock samples.

This work presents an introduction to the definition of a synthetic environment that aims to simulate the core mechanisms of the wave propagation in rock. Such an environment is necessary to study aspects of stress wave interaction with rock masses that are difficult and expensive to investigate in real conditions.

\subsection{Static and dynamic properties of rock}

Hard rock response's under static or quasi-static mechanical loading is well understood, as it has been one of the main areas of research in rock mechanics from its inception. In the more recent years, techniques such as micro-acoustics and computed tomography scans have unveiled the behaviour of the microscopic constituents of rock. Most of these studies have been 
performed in the laboratory but some acoustic emission studies have been performed in situ, allowing the understanding the microfracturation that occurs in rock well before the first visible signs of rock failure.

In what concerns the study of rock dynamic properties, it has been much less intense and started later on, for obvious reasons. Also the relation between laboratory sized cores and real size rock masses under dynamic loads started being studied much later, but it is today gaining momentum due to more recent demands: optimization of blasting, vibration mitigation in mines, urban construction sites and railroads.

Simultaneously, micromechanical models of rock, which have been available for a number of decades (Cundall \& Strack 1979) bloomed with the impulse of the enormous increase of computing power and the gradual enhancement and adaptation of the two and three-dimensional codes to the new hardware, in particular the parallelization of code to modern CPU architecture.

These numerical methods can simulate the richness of macroscopic response of rock without resorting to complex mathematical formulations. The complexity emerges from the interaction of a large number of particles which react to proximity and contact using very simple contact laws. When compared with the finite element or finite difference methods, which are more efficient from a computational point of view, micromechanical methods have the advantage of representing explicitly the imperfections and fractures of rock, allowing a greater interaction and learning between simulation and testing. When it comes to dynamic behaviour, this advantage is even greater, since it is very difficult to grasp what happens inside the rock during high-speed testing.

\subsection{Micromechanical modelling of rock}

The particle element method allows the micromechanical modelling of rock through a densely packed, non-structured, assembly of circular or spherical particles, simulating in two or three-dimensions the basic static and dynamic mechanics of rock behaviour. Discrete element models that employ a time-steeping algorithm are particularly well suited for this task, since they easily perform dynamic and static analysis in the same model, with the same material properties, allowing the inherent response of the material to each environment emerge. Although the characterization of static properties have been reported by several authors, few works, if any exist on the emergent dynamic properties of particle models.

\subsection{Synopsis}

In the first part of this work the generation of the base model and its static properties are described. Then the influence of ball radius and contact stiffness on the elastic constants of the model are studied (Young modulus and Poisson ratio). On the third part the wave propagation test to measure and calculate dynamic elastic properties is described and finally the influence of the DEM model parameters variation on static and dynamic properties is presented and discussed.

\section{METHODOLOGY}

\subsection{Generation of a hard rock bonded particle model}

The characteristics of a micromechanical model can be divided in two groups: one corresponds to the geometry and the second to the microscopic properties of the particles and contacts. The geometry is defined not only by the width and height of the sample, which in this case is rectangular, but also by the statistical size distribution of the particles and their arrangement in a compact assembly.

The procedure adopted to generate the particle assembly (Potyondy \& Cundall 2004) consists of five sequential steps. A closed domain is limited by walls, and filled with a number of particles generated at random positions. The particles are initially generated with half of their final radius. After the insertion of all particles, their radius is expanded gradually and they are allowed to rearrange themselves in a selfbalanced assembly. The average stress in the sample is measured and the particle radii are reduced until the stress state is similar to the desired value, which in this case is close to zero in both directions. Finally, particles touching two or less particles are expanded and allowed to move until they touch at least three neighbours, resulting in a dense and well packed assembly. Then, friction, shear and tension strength is assigned to the contacts between particles that touch or are within a set tolerance and walls delimiting the model sample are deleted. This five-step method builds an assembly of particles with a pre-determined distribution of microproperties. The assembly and micro-properties results in a mechanical response that has to be evaluated through mechanical testing, in this case semi-static compression and wave transmission between the ends of the model.

Since the object of this study is the elastic response of the rock, contacts were not allowed to fail, which was achieved by setting their bond strength to values much higher than those induced by the stress waves, while static Young modulus was calculated in the elastic phase of the model. As to the density, its choice is straightforward, since it is equal to the density of the real rock corrected for the assembly porosity. Contact stiffness is not so easily to assign, since there is no direct way of knowing the resultant macroscopic properties that will result from the model. Several authors (Fakhimi \& Villegas 2007) have worked on this topic and suggested calibration methods, both in the form of calibration curves, which still demand some finetuning, or directed trial-and-error. This method can be time consuming if the model is large. Since the models in this study were relatively small and the authors experienced in this procedure, calibration was quite easy, and the desired macroscopic response was attained in less than four iterations. In each of these iterations, the 

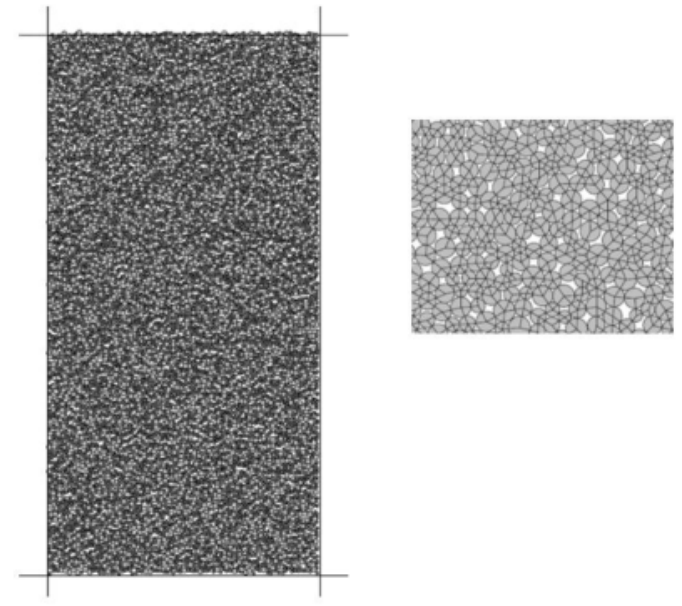

Figure 1. Image of the rock core model and detail showing the connections between balls.

following procedure was followed. First, contact bond strength is set to very high values, so that an elastic response is obtained during the calibration; then, the normal contact stiffness $K_{n}$ and shear contact stiffness $K_{s}$ are set to initial values, which can be obtained from a previous calculation or from parametric curves. A uniaxial compression test of the sample is done and a Young's modulus is obtained. $\mathrm{K}_{\mathrm{n}}$ is then adjusted to increase or decrease until an acceptable match of the Young's modulus is attained, and during this adjustment Ks varies in the same proportion as $K_{n}$. The Poisson ratio is then adjusted to the desired value by keeping $\mathrm{K}_{\mathrm{n}}$ constant while $\mathrm{K}_{\mathrm{s}}$ is changed, since Poisson modulus is related to the $\mathrm{K}_{\mathrm{n}} / \mathrm{K}_{\mathrm{s}}$ ratio. At the end of this step both Young and Poisson moduli are set and the calibration is finished.

The reference model in this study is $50 \mathrm{~mm}$ wide and $100 \mathrm{~mm}$ tall, the minimum ball radius is $0.25 \mathrm{~mm}$ and the maximum is $0.415 \mathrm{~mm}, 1.66$ times larger. Particle density was set to $2941 \mathrm{~kg} / \mathrm{m}^{3}$. The normal to shear contact stiffness ratio is 2.5 and the normal stiffness is equal to $40 \times 10^{9} \mathrm{~Pa}$.

Two synthetic samples generated with the same sequence of commands have different responses, since there is randomness in the particle arrangement, hence the contact networks will be different. This is similar to the response of rock, where samples taken from the same core have slightly different properties.

In the current case the authors didn't aim to reproduce a previously defined macroscopic behaviour, but to find out how the same set of micro-properties and their variation results in terms of static and dynamic behaviour.

\subsection{Static testing and properties}

Figure 2 shows the plot of normal stress against vertical deformation in an unconfined compression test. The pictures in the plot show the forces between particles in

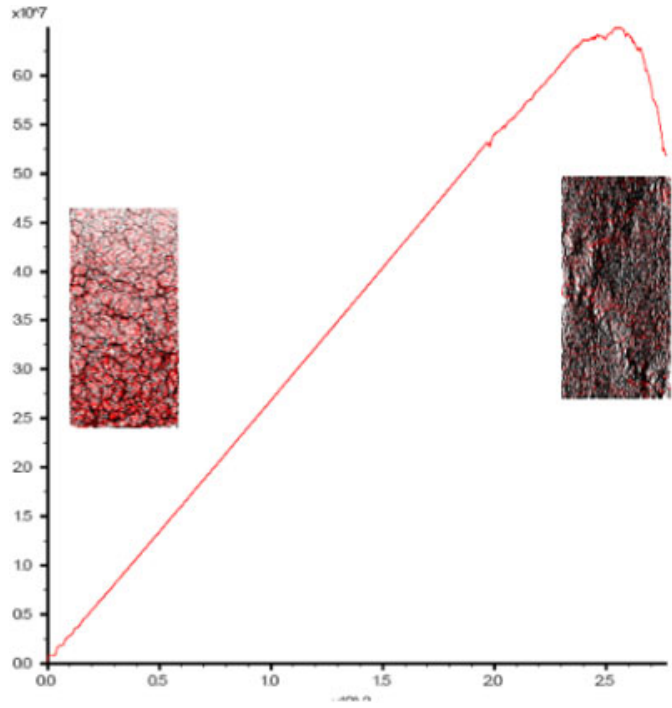

Figure 2. Plot of force-displacement curve in unconfined compressive test until failure and superposition of contact forces in the model at start and end of the simulation (red line denotes tension and black lines compression). Notice the initial effect of self-weight (left insert figure) and the final pattern of forces showing the formation of diagonal macro-fracture in the rock. Forces on the right are many times larger than on the left.

the beginning and end of the simulation. At the beginning of the test, the predominant stress is due to the self-weight of the model. At the end, the vertical compressive stress is predominant and shear cracks are completely defined.

Young and Poisson moduli are calculated in a similar fashion as a laboratory test, by considering the normal stress and the associated vertical and horizontal deformations, measured between the ends of the model or in its interior. The elastic parameters were calculated for the reference model and thirty other models where one of the following parameters was changed: ball radius, ball radius ratio (ratio between maximum and minimum ball dimensions), contact normal stiffness and ratio of normal and shear contact stiffness.

\subsection{Dynamic testing and properties}

After the generation, calibration and static testing of each model, it is tested for dynamic properties. This test is similar to a laboratory Ultra-Sound test: a wave is generated in the bottom boundary and is received in the opposite wall. The frequency of the wave was picked up so that the wave is preserved while travelling across the model, according to previous work by Resende et al. (2010).

The distance between the ends of the model is then divided by the time lapse between generation and wave arrival. Both shear and compressive waves can be generated, although there are specific difficulties to each type of wave. Figure 3 show the location 


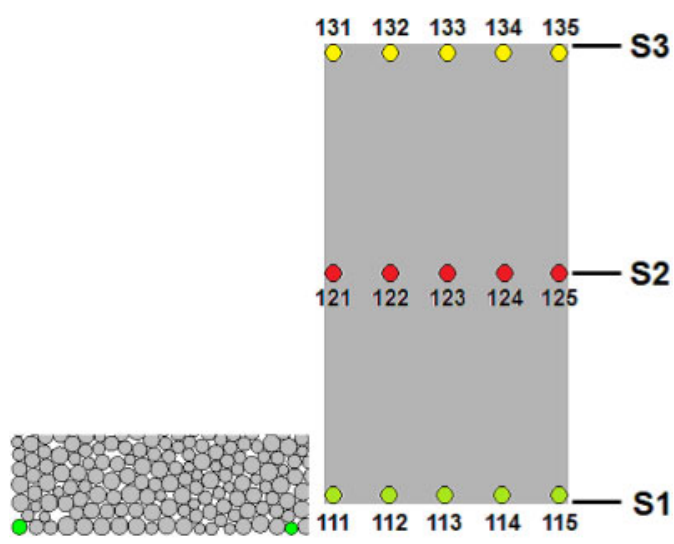

Figure 3. Location of the probes where particle velocity was extracted and detail of the model with identification of the precise probe particles' locations.

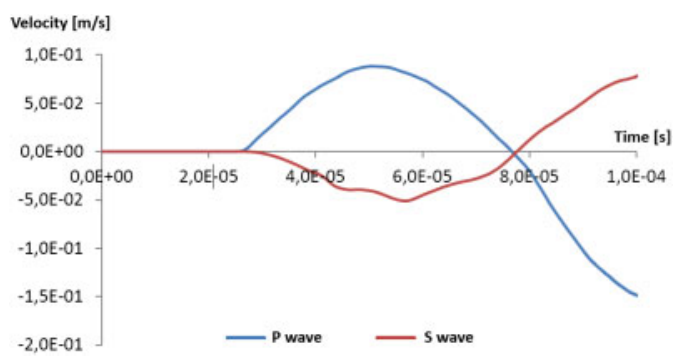

Figure 4. Particle velocity plot at section S3 for identification of compressive and shear wave arrival time.

of the probed particles. In each section five particles were selected and their velocity time-histories were analysed.

Figure 4 shows the vertical and horizontal particle velocities extracted from a calculation where a compressive and a shear wave were injected at the bottom of the model. The vertical velocity and horizontal velocities were then measured at the top of the model and analysed to determine wave arrival time. It is clear from the analysis of the two plots that it is easier to identify compressive wave arrival, since shear waves are prone to contamination from the faster $\mathrm{P}$ wave. It is interesting to note that the same problem is recurrent in laboratory testing of shear wave velocity, and the same strategies for wave identification are applied here.

Wave phase velocity $V_{P}$ or $V_{S}$ is then calculated from dynamic test results using:

$V=\frac{L}{t}$

where $L$ is the distance between the actuator and the sensor and $t$ is the time interval between wave generation and wave arrival.
As shear and compressive wave velocities are determined in the model, both dynamic Young's modulus $\mathrm{E}_{\text {dyn }}$ and Poisson coefficient $v_{\text {dyn }}$ can be calculated. If Poisson coefficient is calculated first,

$$
v_{d y n}=\frac{\left(\frac{V_{P}}{V_{S}}\right)^{2}-2}{2 \cdot\left(\frac{V_{P}}{V_{S}}\right)^{2}-2}
$$

Then using rock density $\rho$, Young's modulus becomes:

$E_{d y n}=\rho \cdot V_{P}{ }^{2} \cdot \frac{\left(1+v_{d y n}\right)\left(1-2 \cdot v_{d y n}\right)}{1-v_{d y n}}$

\section{RESULTS}

\subsection{Geometric properties: ball radius and radius ratio}

Figure 5 shows the evolution of both static and dynamic properties when the ball size varies. The first observation is that dynamic properties are always higher than static properties. This is apparent in the majority of rock materials and is extensively documented (Barton 2007). This has also been reported in previous tests with particle models of rock. The particle models simulate the real mechanics of particle adjustment under static load and the wave propagation from particle to particle.

The variation of particle size, while keeping the particle radius ratio (larger particle diameter over smaller particle diameter) constant does not change the elastic parameters significantly. It is interesting to notice that both in the Young and Poisson moduli the difference between static and dynamic properties seems to diminish with higher ball size.

Difference between static and dynamic properties also decreases when the ball size ratio increases, as observed in Figure 6. But here, a more interesting effect is evident: when all particles are of the same size, i.e. the particle size ratio is equal to one, there is smaller complexity of the particle assembly and both static and dynamic Young's modulus increases abruptly. It should be noted that PFC manuals advise the use a value of $R_{\max } / R_{\min }$ of 1.66 for rock material.

\subsection{Normal contact stiffness and ratio of shear and contact stiffness}

Figure 7 confirms an intuitive tendency: as the normal and shear contact stiffness increase, the global stiffness of the model does to. This is well known, but it is interesting to see what happens in the dynamic case. It also shows that if normal stiffness is constant and shear stiffness decreases (making the $\mathrm{K}_{\mathrm{n}} / \mathrm{K}_{\mathrm{s}}$ ratio grow) global stiffness of the model decreases, but the 


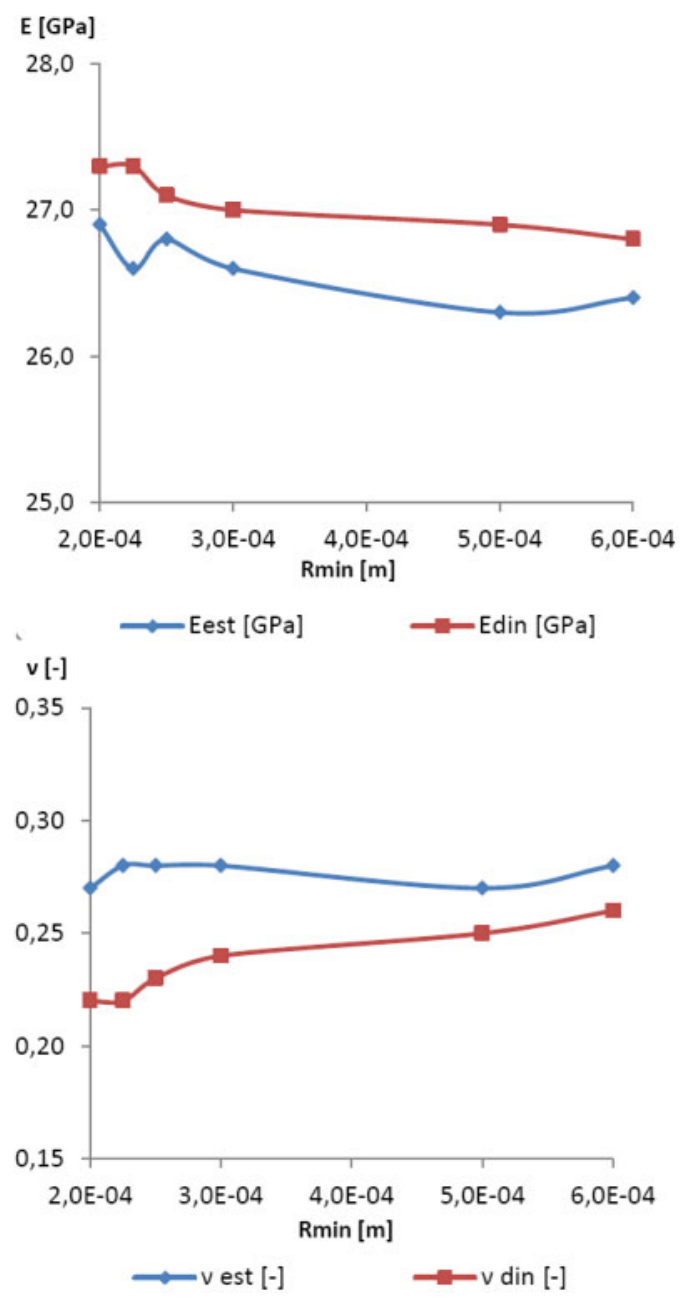

Figure 5. Evolution of static and dynamic Young's modulus (top) and Poisson's ratio (bottom) with ball radius.

decrease here is not linear, indicating that even if shear stiffness is very low, the normal stiffness assures the "skeleton" of the rock.

It is interesting to note what happens to Poisson ratio. When the contact stiffness increases (Figure 8) the Poisson ratio variation is small. But when the contact stiffness ratio increases, that is, normal stiffness is more important than shear stiffness, the Poisson modulus increases because the lateral movement of the particles becomes less constrained (Figure 9).

\subsection{Dynamic properties}

Figures 10 shows the evolution of P-wave velocity with contact stiffness and contact stiffness ratio (S-wave evolution is similar to P-wave's and is not shown here for lack of space).

Stress waves are faster when normal contact stiffness increases slow down as the stiffness ratio

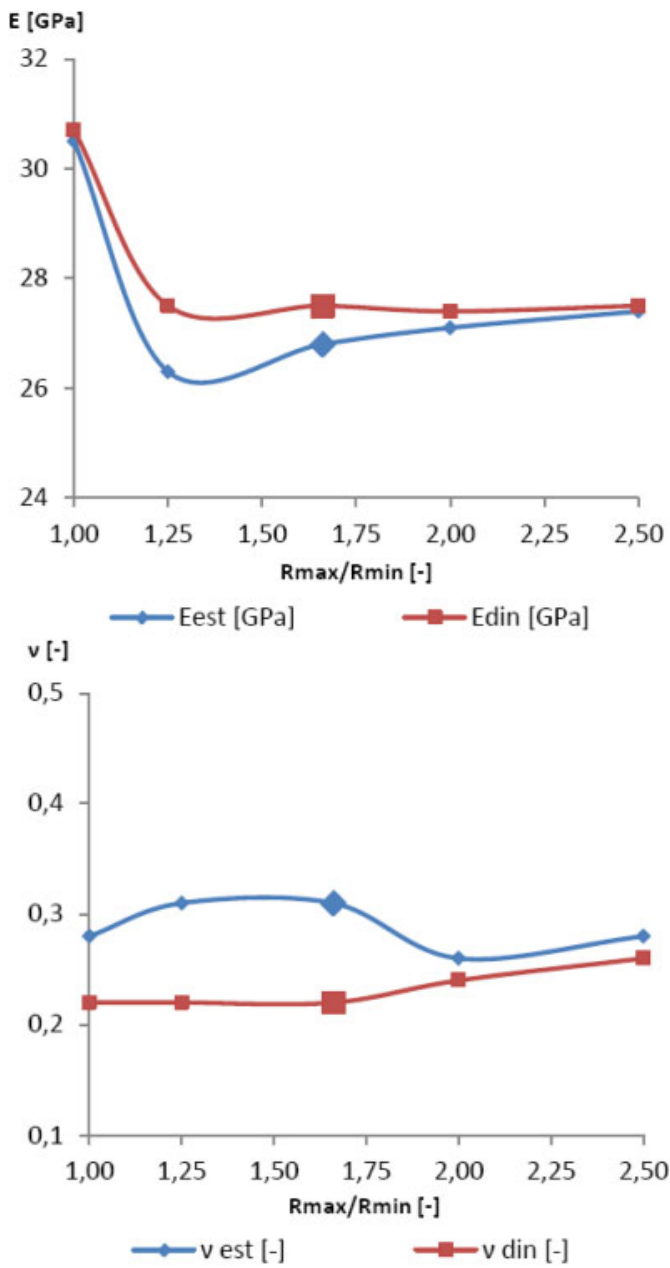

Figure 6. Evolution of static and dynamic Young's modulus (top) and Poisson's ratio (bottom) with ball radius ratio.

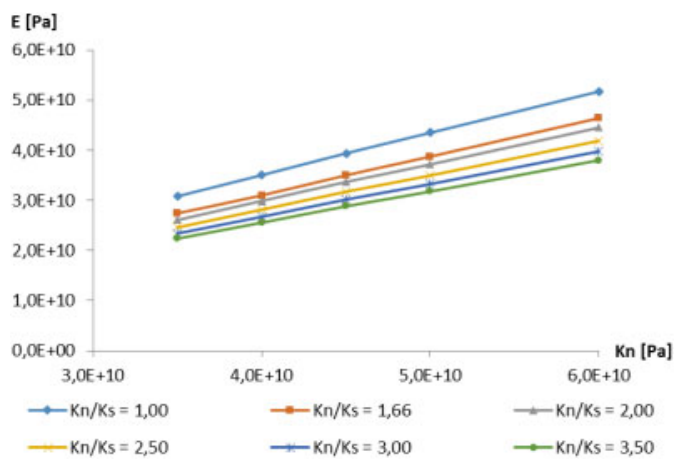

Figure 7. Evolution of static Young modulus with contact stiffness and contact stiffness ratio.

decrease, and both get faster as the stiffness increases. But to find out what happens to elastic constants, the influence of both waves must be considered through equations 2 and 3 and compiled in next section. 


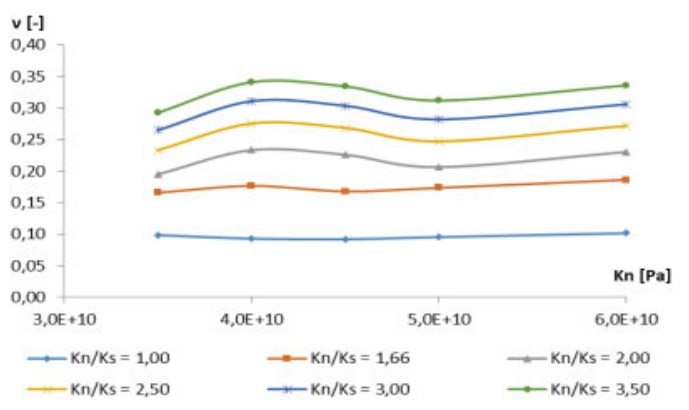

Figure 8. Evolution of static Poisson modulus with normal contact stiffness.

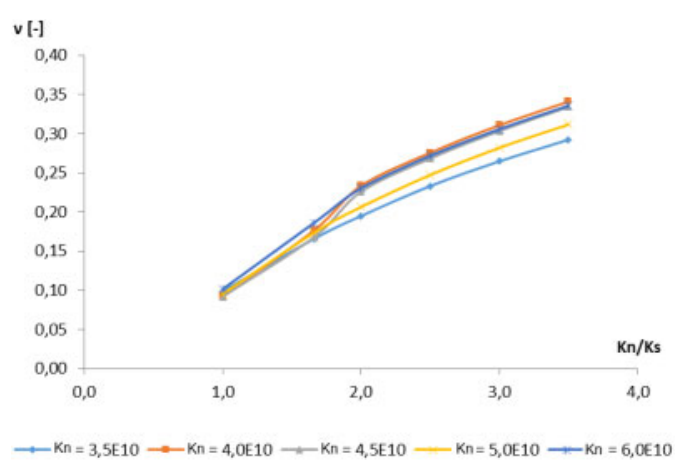

Figure 9. Evolution of static Poisson modulus with contact stiffness ratio.

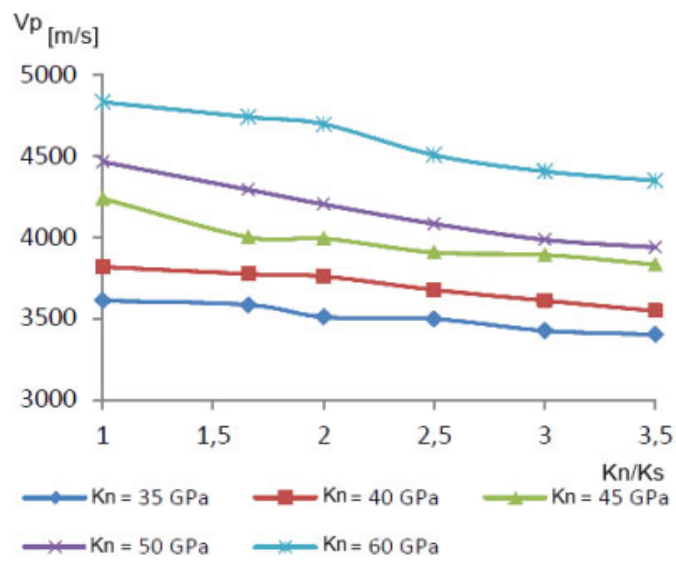

Figure 10. Evolution of compressive wave velocity with contact stiffness ratio.

\subsection{Comparison of static and dynamic properties}

Finally, we compare, in Figure 11, the static and dynamic properties for a constant contact normal stiffness $\left(\mathrm{K}_{\mathrm{n}}\right)$ of $60 \mathrm{GPa}$. Two observations are important. First, the major tendencies are similar in both Young and Poisson modulus for static and dynamic elastic parameters. Second, the dynamic Young modulus is larger than the static, but only when $\mathrm{K}_{\mathrm{n}} / \mathrm{K}_{\mathrm{s}}$ is

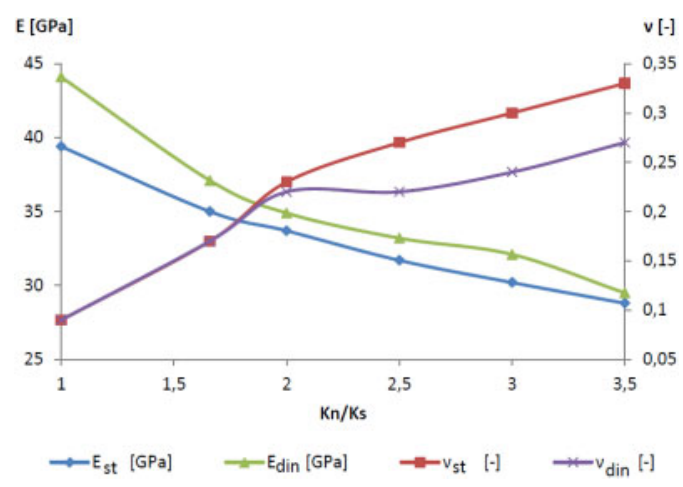

Figure 11. Evolution of static and dynamic elastic properties with contact stiffness ratio.

larger than 2.0. It is useful to notice that Potyondy \& Cundall (2004) suggest that to simulate static behaviour of hard rock, the contact stiffness ratio should be above this value.

\section{CONCLUSIONS}

This paper presented a comparative investigation on the static and dynamic elastic macroscopic response of Bonded Particle Models of hard rock cores.

The study of static behaviour of this class of models is well advanced, but the dynamic range is still in its start. The authors think that particle models will be intensely used on more complex environments such as dynamic, thermo-hydraulic-mechanical coupled analysis, fractured rock masses, etc.

The tendencies found in this study confirm that the elastic dynamic response of bonded particle models follows the patterns exhibited in experimental investigations, paving the way for further use of these techniques in more complex applications.

\section{REFERENCES}

Andrade, C. 2012. Modelação do Comportamento Estático e Dinâmico da Rocha com Modelos de Partículas. MSc thesis, Porto University.

Cundall, P. A. \& Strack, O. D. L. 1979. A discrete numerical model for granular assemblies. Geotechnique 29(1): 47-65.

Barton, N 2007. Rock Quality, Seismic Velocity, Attenuation and Anisotropy. Taylor and Francis.

Fakhimi, A. \& Villegas T 2007. Application of dimensional analysis in calibration of a discrete element model for rock deformation and fracture. Rock Mechanics and Rock Engineering 40(2): 193-211.

Potyondy, D. O. \& Cundall, P. A. 2004. A bonded-particle model for rock. International Journal of Rock Mechanics and Mining Sciences 41(8): 1329-1364.

R Resende, LN Lamas, JV Lemos, R Calçada 2010, Micromechanical modelling of stress waves in rock and rock fractures. Rock Mechanics and Rock Engineering 43(6): 741-761. 